Regards sur l'économie allemande

Bulletin économique du CIRAC

97 | 2010

Varia

\title{
Les élites économiques sont-elles encore si différentes en France et en Allemagne?
}

Joël Massol, Thomas Vallée et Thomas Koch

\section{CpenEdition}

Édition électronique

URL : http://journals.openedition.org/rea/4104

DOI : $10.4000 /$ rea.4104

ISBN : 978-2-8218-0890-4

ISSN : 1965-0787

\section{Éditeur}

CIRAC

Édition imprimée

Date de publication : 1 juillet 2010

Pagination : 5-14

ISSN : 1156-8992

\section{Référence électronique}

Joël Massol, Thomas Vallée et Thomas Koch, «Les élites économiques sont-elles encore si différentes en France et en Allemagne? », Regards sur l'économie allemande [En ligne], 97 | juillet 2010 mis en ligne le 01 juillet 2012, consulté le 04 mai 2019. URL : http://journals.openedition.org/rea/4104 ; DOI : 10.4000/rea.4104 


\title{
Les élites économiques sont-elles encore si différentes en France et en Allemagne?
}

\author{
Joël Massol, Thomas Vallée, Thomas Koch
}

En France comme en Allemagne, les dirigeants des grandes entreprises font régulièrement la une de l'actualité ou de la presse, spécialisée ou non. Tout citoyen peut ainsi découvrir le montant exorbitant de leurs rémunérations ou de leurs 'parachutes dorés', les victoires ou déboires de tel ou tel grand patron. Mais que sait-on vraiment des formations, des parcours professionnels de ceux qui président aux destinées des entreprises figurant parmi les plus grands groupes mondiaux ? L'image des élites économiques dans les sociétés française et allemande relève encore trop souvent de la mythologie ou de la caricature.

Pourtant, nombreux sont les travaux qui, en France et en Allemagne, mais aussi dans d'autres pays, portent sur les élites économiques, notamment leur production et reproduction, leur profil sociologique. Tous ou presque constatent que les équipes managériales s'insèrent dans des cultures nationales particulières qui les rendent foncièrement différentes, et les managers allemands et français ne semblent pas faire exception à la règle.

Or la globalisation des économies a imposé des bouleversements inédits. Jamais les grandes entreprises n'ont autant fait fi des barrières nationales, jamais leurs activités n'ont atteint de tels degrés d'ouverture et d'interpénétration, favorisés par la libéralisation presque totale des mouvements de capitaux et des hommes. Les grandes firmes multinationales, et aussi celles dont l'origine historique est allemande ou française, se sont internationalisées au pas de charge et adaptées à cette intégration mondiale. Mais aujourd'hui, alors que près de la moitié de leur capital est détenue par des investisseurs étrangers, alors qu'elles adoptent les standards anglo-saxons de comptabilité et les mêmes outils de gestion, souvent la même langue de travail que leurs homologues américaines, que reste-t-il des particularités nationales de leur management? Les systèmes nationaux de formation des élites françaises et allemandes se sont-ils appuyés sur leurs atouts pour continuer de former des élites économiques dont la diversité et les spécificités constituaient la richesse, ou au contraire pour faciliter une harmonisation sur le modèle anglo-saxon?

\section{Des élites économiques à la fois différentes et semblables}

Comme tout système de règles, celui de la formation et du recrutement des élites s'enracine et évolue à partir des traditions, dans des cadres juridiques et institutionnels qui varient selon les pays, les aires géographiques et culturelles (Hofstede, 1980 ; D'Iribarne, 1989 ; Suleiman/Mendras, 1995). II n'est donc a priori pas surprenant de constater que les différences l'emportent sur les ressemblances dans les comparaisons et études empiriques qui ont été menées jusqu'alors sur les formations et les profils des dirigeants français et allemands (Bauer/BertinMourot, 1996 ; Joly, 2005 ; Hartmann, 2007).

En France, l'université est nolens volens à l'écart de la formation des élites économiques et politiques. Cette formation paraît confisquée par les grandes écoles, véritable voie royale vers les fonctions de dirigeants et qui consacre ainsi la "tyrannie du diplôme initial » (Bauer/Bertin-Mourot, 1995). De manière générale, que ce soit dans la haute administration ou dans les sphères politique ou écono-

En France, tyrannie du diplôme d'une grande école 
Sélection précoce des élites françaises

Exception française : le parachutage

Des élites ou une élite? mique, les dirigeants français sont formés dans des grandes écoles telles que I'ENA, l'Ecole Polytechnique, Ponts et Chaussées, HEC, les ENS, Sciences Po. Selon Joly (2005), environ $70 \%$ des directeurs de cabinets ministériels étaient des énarques dans les années 1990 ; 14 sur 18 Premiers ministres de la V Vépublique étaient diplômés des grandes écoles et/ou membres des grands corps de l'Etat (Mines, Ponts, Inspection des finances, Cour des comptes et Conseil d'Etat). C'était aussi le cas d'environ $60 \%$ des ministres du gouvernement Jospin, de la moitié des ministres du gouvernement de Villepin. En 2005, 62 \% des PDG des 100 premiers groupes français étaient issus de grandes écoles.

Le développement important des universités de masse depuis les années 1960 a peu affecté la suprématie des grandes écoles, en dépit de leurs faibles effectifs d'étudiants : au début des années 2000, l'ENA produisait environ 100 diplômés par an, l'Ecole Polytechnique et HEC environ 400 (Hartmann, 2007). Cette stabilité de la sélection s'accompagne d'une précocité qui ne manque pas d'étonner les observateurs étrangers. Une fois l'étudiant de classe préparatoire " admis » dans une grande école, soit deux ou trois ans après le bac, à l'âge de 20 ou 21 ans, son avenir en tant que dirigeant dans le domaine administratif, politique ou économique est assuré - un énarque qui a réussi le concours externe commence en général sa carrière à l'âge de 25 ou 26 ans.

Une autre particularité française est constituée par la migration des élites politicoadministratives issues de l'ENA et des grands corps de l'Etat vers les postes de direction des grandes entreprises privées françaises. Le phénomène a fait lui aussi l'objet de nombreux travaux (Suleiman/Mendras, 1995 ; Joly, 2005b). Le cas de Daniel Bouton constitue un exemple emblématique. Issu de l'ENA et de l'Inspection des Finances, il est à 38 ans directeur du Budget au ministère de l'Economie et des Finances. En 1991, il passe à la Société Générale, avant d'en devenir, seulement 2 ans plus tard, le directeur général. Ce parachutage ou ce 'pantouflage', puisqu'il s'agit en l'occurrence du passage d'un membre d'un grand corps de l'Etat à l'entreprise privée, est loin d'être un cas isolé (Hartmann, 2007). II pose problème à plus d'un titre, et en premier lieu parce que la vocation déclarée de l'ENA est de former des cadres pour la haute fonction publique et non de servir de tremplin vers les fonctions de dirigeants des grandes entreprises privées (cela concernait $11 \%$ des grands patrons français). Ce parachutage constitue en outre un frein puissant à la promotion interne et dévalorise les carrières longues en entreprise : les 'parachutés' bloquent la carrière de ceux qui ont travaillé depuis longtemps et ont fait leurs preuves au sein de l'entreprise. Seuls $8 \%$ des PDG des 200 premiers groupes français avaient fait une 'carrière-maison' en 1995 (Bauer/Bertin-Mourot, 1996). Des travaux récents montrent que cette «conversion de la noblesse d'Etat en aristocratie des affaires " (Dudouet/ Grémont, 2007) a été largement favorisée par les privatisations des grands groupes français, et aussi que ces anciens commis de l'Etat dominent le monde des grands patrons par l'importance économique des entreprises qui les ont accueillis comme par leurs réseaux d'influence. Et cette prééminence du pôle politico-administratif ne disparaît pas avec la fin des privatisations : même pendant la période 2002-2006, le recrutement des numéro 1 des quarante premières capitalisations boursières voit les dirigeants issus de la haute fonction publique (40\%) arriver devant les dirigeants issus de l'entreprise (38\% ; Dudouet/Grémont, 2007).

Bourdieu a été l'un des premiers à mettre en évidence le fait que le système de formation des grandes écoles, fondé à l'origine pour créer une méritocratie républicaine, produisait en réalité une classe dirigeante largement homogène, dans laquelle les classes moyennes et populaires, tout comme les personnes issues de l'immigration, étaient fortement sous-représentées. Dans son ouvrage "La distinction », il avait notamment souligné à quel point le capital culturel produit par la formation élitiste et l'habitus de la bourgeoisie et de la grande bourgeoisie étaient déterminants pour la constitution des élites. Malgré quelques récentes expérimentations de démocratisation, force est de constater la grande stabilité du modèle français dans le temps. Ce modèle de formation des élites, que ces der- 
nières soient politiques, administratives ou économiques, semble toujours se caractériser par une fermeture sociale liée à une approche méritocratique exclusivement scolaire et de plus en plus inégalitaire (van Zanten, 2008). L'homogénéité du système de formation et de l'appartenance sociale des élites françaises est même si forte qu'il serait peut-être plus pertinent d'utiliser le concept d'élite au singulier, contrairement à la plupart des autres pays, dont l'Allemagne.

En Allemagne, la formation des élites économiques n'est pas le domaine réservé de quelques établissements privilégiés comme en France. II existe aujourd'hui certes quelques écoles supérieures de commerce privées, prestigieuses et chères, mais la formation des dirigeants des grandes entreprises se déroule traditionnellement dans les universités publiques: dans les facultés des sciences, des sciences économiques et de gestion, de droit, et dans les départements technologiques. A côté des universités et des universités techniques, les Fachhochschulen (équivalent des IUT), avec leurs formations plus courtes d'un an et plus axées sur la formation pratique, jouent également un rôle dans la formation des cadres économiques, même si c'est davantage au niveau des cadres supérieurs que des membres des comités exécutifs et des directoires. Les études menées sur les dirigeants des grandes entreprises montrent que, depuis 1955, il n'existe pas d'université qui constituerait un vivier privilégié pour les carrières de dirigeants. II n'existe donc pas davantage de hiérarchie établie des établissements de formation (Hartmann, 2005).

Cette grande diversité des origines géographiques et des formations est aux antipodes de l'homogénéité française. Les dirigeants allemands sont juristes, économistes, mathématiciens de formation, ou ingénieurs en chimie, en construction mécanique. Même si cela ne constitue pas la règle, on a vu, dans un passé plus ou moins récent, plusieurs docteurs de musicologie ou de philosophie accéder au poste de président du directoire ou du conseil de surveillance de très grandes entreprises. Daniel Gœudevert, vice-président de WW aux côtés de Ferdinand Piëch au début des années 1990, n'était-il pas germaniste de formation avant d'être nommé président du directoire de Ford-Allemagne? Si on examine de plus près le contenu des formations des dirigeants allemands, on est frappé de constater leur niveau de spécialisation, surtout comparé à leurs homologues français. En France, les filières d'excellence, qu'elles soient des métiers de l'ingénieur ou de la haute fonction publique, forment essentiellement des 'généralistes' préparés aux postes d'encadrement technique ou administratif. Dans de nombreuses filières allemandes, notamment celles des métiers de l'ingénieur, l'aboutissement des études supérieures demeure traditionnellement le doctorat, ce qui implique une spécialisation dans un domaine de la recherche et donc un rapport plus érudit aux savoirs, et souvent une pensée spéculative, ou au moins critique.

Les sociétés allemandes adoptent, comme leurs homologues françaises, très largement les hiérarchies introduites par l'enseignement supérieur. Elles recherchent aussi un niveau d'excellence académique, mais par la force des choses, leur sélection est sensiblement plus tardive. L'âge moyen d'un diplômé est en effet d'environ 28 ans, ce qui signifie que la sélection a lieu en Allemagne environ 7 à 8 ans plus tard qu'en France. De plus, ce critère de sélection n'est ni unique ni exclusif. L'apprentissage continue à jouer un rôle non négligeable et plus important qu'en France - il offre une chance sérieuse pour la promotion dans l'entreprise, y compris dans les professions techniques et commerciales : $27 \%$ des patrons allemands des 'Top 200' dans les années 1990 sont passés par l'apprentissage, et nombreux sont ceux qui parmi eux ont poursuivi leurs études pour aller jusqu'au doctorat. C'est ainsi que la part des dirigeants ayant commencé leur carrière au sein de l'entreprise est traditionnellement plus importante en Allemagne qu'en France : $32 \%$ des dirigeants des 'Top 200' allemands au début des années 1990 contre $6 \%$ en France (Bauer/Bertin-Mourot, 1996).

De plus, et c'est là une autre différence avec le modèle français, il n'existe pratiquement aucune passerelle professionnelle entre les secteurs de l'administration, de la politique et du secteur privé : en 2006, un seul numéro 1 des 100 premiers
En Allemagne, pas de voie royale...

... mais une grande diversité des parcours et des spécialités

Légitimité académique, mais aussi apprentissage et expérience professionnelle

Pas de passerelle entre public et privé 
Une sélection sociale décisive : les limites de la méritocratie groupes allemands avait fait une 'incursion' dans un autre secteur d'activité, la vie politique, avant d'entrer dans le secteur privé : l'ex-ministre de l'Economie Werner Müller. Cette absence de mobilité sectorielle ou fonctionnelle s'avère d'ailleurs une constante allemande : au début des années 1990, seuls 3,5\% des dirigeants des 'Top 200' allemandes venaient de l'administration, contre $44 \%$ en France.

Malgré la diversité des origines géographiques et des établissements de formation, la variété des diplômes et des voies d'accès aux fonctions de direction des grandes entreprises, les élites économiques allemandes se caractérisent par la même homogénéité sociale qu'en France. Hartmann a mis en évidence que les chances d'obtenir une place à la direction d'une grande entreprise allemande sont 10 fois supérieures pour le fils d'un cadre supérieur que pour celui d'un ouvrier, avec un niveau universitaire identique : le doctorat. Et il ajoute que cette discrimination sociale, loin de diminuer, s'accroît avec le temps.

Bien qu'il n'y ait pas comme en France de "tyrannie du diplôme initial » ou de sélection sociale institutionnalisée, les procédures et mécanismes de sélection et de 'fabrication' des élites produisent en Allemagne des résultats similaires dans les deux pays quand on les considère du point du vue sociologique. On constate en effet la prépondérance des milieux aisés et de la grande bourgeoisie (une fraction qui représente moins de $5 \%$ de la société) dans les élites économiques qui, de ce fait, apparaissent beaucoup plus comme des classes 'dominantes' ou des 'élites de pouvoir' que comme des élites méritocratiques et fonctionnelles.

Pour Hartmann, qui suit ici Bourdieu, la réussite universitaire est une condition nécessaire mais pas suffisante. Ce qui est décisif en dernière instance, ce n'est pas les études ou les performances professionnelles - qui caractérisent aussi bien le système allemand que le système français -, c'est la sélection par l'habitus 'bourgeois' (confiance en soi, culture générale...). Cette sélection sociale intervient en France au moment des concours de recrutement des grandes écoles (notamment des épreuves orales), et en Allemagne au moment des entretiens d'embauche des cadres supérieurs et dirigeants dans les grandes entreprises. II est vrai que le discours méritocratique n'est pas identique en Allemagne et en France. En Allemagne, il concerne davantage la performance dans l'entreprise que les résultats scolaires et les études, mais il produit quoi qu'il en soit le même " mythe » (cf. le titre de l'ouvrage principal de Hartmann: "Der Mythos von den Leistungseliten ») destiné à occulter la réalité sociale des rapports de pouvoir et des privilèges économiques.

\section{La globalisation : de puissants courants d'homogénéisation des situations nationales}

Aussi différents soient-ils, ces modèles nationaux de formation des élites économiques en France et en Allemagne sont aujourd'hui exposés à de profondes mutations de leur environnement social et économique qui estompent certaines particularités nationales et modifient les structures du pouvoir économique. Ces changements concernent principalement l'internationalisation des firmes dans la globalisation, et l'alignement sur le modèle anglo-saxon des codes de gouvernance (Massol/Vallée, 2008) et des cultures d'entreprise (Reisach, 2007).

II existe de nombreuses manières de mesurer l'internationalisation des firmes. Parmi ces différentes approches, l'indice de transnationalité (ITN) qui est publié chaque année par la CNUCED depuis 1995 et prend notamment en compte la part des actifs situés à l'étranger, la part des emplois implantés à l'étranger et la part du CA réalisé à l'étranger, est l'un des moins controversés (CNUCED, 2008). La comparaison des indices de transnationalité au niveau mondial met en évidence que les firmes françaises et allemandes jouent un rôle de tout premier plan dans la mondialisation. On constate leur forte présence dans la liste des 100 premières sociétés transnationales (STN) mondiales non financières en 2006 : elles sont 29 au total (dont 13 cotées au DAX 30 et 13 cotées au CAC 40) ; il s'agit entre autres, dans l'ordre décroissant, de : Linde, Pernod Ricard, Lafarge, Total, 
Saint-Gobain, Christian Dior, Suez, Siemens, Sanofi-Aventis. II est en outre à noter que leur nombre s'est considérablement accru : en 1995, elles n'étaient que 20 à figurer parmi les 100 premières mondiales en 1995 (CNUCED, 1997).

Les évolutions sont analogues dans le groupe des sociétés financières allemandes et françaises. Parmi les 50 premières STN financières mondiales en 2006, on peut dénombrer au total 10 entreprises françaises (6) et allemandes (4) dont 9 cotées au CAC 40 ou au DAX 30 qui se caractérisent par un ITN particulièrement élevé : Allianz, BNP Paribas, Axa, Société Générale, Deutsche Bank, Crédit Agricole, Natexis Banque Populaire, Dexia, Commerzbank, DZ Bank. Elles ont en outre sensiblement amélioré leur position dans le classement mondial depuis 1999 et même par rapport à l'année précédente.

Cette internationalisation croissante des grandes entreprises est renforcée par des changements importants dans l'environnement économique des firmes. Dès le milieu des années 1980, on assiste en effet, en France comme en Allemagne, à l'apparition d'un contexte propice à la montée en puissance d'investisseurs institutionnels et étrangers (hedge funds, private equity). En France, les vagues de privatisation (1986-1988, puis 1993-1999), la déréglementation des marchés financiers et les nombreuses augmentations de capital ont fortement favorisé une montée rapide des fonds étrangers, souvent nord-américains, dans le capital des grandes entreprises françaises (Massol/Vallée, 2008). En Allemagne, une des caractéristiques majeures du capitalisme rhénan s'estompe entre 1995 et 2005 : la 'banque-maison' (Hausbank) cède sa place d'actionnaire de référence au profit des fonds d'investissement. En une décennie, le nombre de familles, de particuliers ou de fondateurs qui étaient actionnaire principal dans les 100 plus grandes entreprises cotées baisse de $23 \%$, celui des banques de $50 \%$, tandis que le nombre des fonds de gestion collective qui deviennent actionnaire de référence augmente de $47 \%$. Comme en France, la part des non-résidents dans la détention du capital des entreprises du DAX 30 augmente considérablement depuis la fin des années 1990. Certaines entreprises-phares du DAX 30 ont un actionnaire de référence qui est un fonds d'investissement étranger : en 2005, Infineon est détenu à hauteur de 18,23\% par Wachovia Trust Company, tandis qu'Alliance Capital Management détient $11,73 \%$ de Continental.

Grâce à leur nouvelle position de force, les investisseurs institutionnels étrangers ont pu imposer leurs conceptions communes du gouvernement d'entreprise. Leurs exigences ont eu d'autant plus de poids qu'elles étaient aussi promues par les agences de notation et les analystes financiers internationaux. Elles ont fortement contribué, par exemple, dans les années 1990, à l'adoption d'un référentiel comptable international qui permette de comparer les entreprises de différentes nationalités sur la base d'une information homogène et normalisée. II s'agit en réalité de deux référentiels anglo-saxons : US GAAP (US Generally Accepted Accounting Principles) et IASC (International Accounting Standards Committee). Ces transformations sont liées à l'internationalisation des firmes, mais aussi à la nécessité de renforcer le contrôle des managers après une succession de scandales et de faillites retentissants de grandes entreprises (Holzmann, Mannesmann, Crédit Lyonnais, Vivendi Universal...) ; cela a donné lieu au sein des différents pays de l'OCDE à l'adoption de codes de bonne gouvernance qui frappent par leur ressemblance (Massol/Vallée, 2008).

Dans ce contexte de mondialisation et d'internationalisation des firmes, les mutations qui modifient en profondeur les conditions de pilotage des grandes entreprises ne laissent pas intacts les modèles nationaux de culture d'entreprise, au point que le modèle allemand paraît tout à coup comme frappé d'obsolescence en tombant dans "le piège de l'américanisation" (Reisach, 2007). Dans le " combat des cultures », pour reprendre le titre de l'ouvrage d'Abelshauser (2003), le modèle shareholder anglo-saxon paraît remporter une victoire décisive sur le modèle stakeholder auquel se rattachent en premier lieu le modèle allemand, et le modèle français dans une moindre mesure. Face aux diktats de la shareholder value et du court-termisme nord-américain, nombreuses sont les
Montée en puissance des investisseurs institutionnels et étrangers...

... et diffusion de
référentiels anglo-saxons

Des cultures d'entreprise menacées : shareholder value versus stakeholder value 
Internationalisation de la formation des équipes dirigeantes

grandes entreprises allemandes et françaises qui s'exécutent et adoptent la publication trimestrielle de leurs résultats, le retour rapide sur investissement comme priorité de gestion, la langue anglaise comme langue de travail ou une approche trop exclusivement financière du développement de l'entreprise (Reisach, 2007).

\section{Profils et parcours des dirigeants des grandes entreprises du CAC 40 et du DAX 30 : continuités et ruptures}

Sous l'influence de ces mutations, le mouvement d'internationalisation des formations déjà mis en évidence au début des années 1980 s'est amplifié en se généralisant. En France, le développement d'un marché international des formations universitaires est intimement lié à l'internationalisation des grandes écoles et à une conception de l'excellence qui valorise l'ouverture sur l'étranger autant que le « culte de la performance », pour reprendre le titre de l'ouvrage d'Alain Ehrenberg (1991), et le goût du risque. Les élèves étrangers constituent à présent un quart des effectifs de l'Ecole Polytechnique ou de HEC (Wagner, 2008). L'Allemagne n'échappe pas à ces nouvelles règles plus ou moins tacites qui jalonnent le parcours initiatique des futurs managers : de plus en plus d'étudiants, en particulier ceux qui sont inscrits dans des facultés d'économie ou de gestion, partent de préférence en Amérique du Nord pour obtenir un MBA, véritables 'lettres de noblesse' qui leur apporteront une forme de consécration de leur excellence nationale tout en attestant leur capacité à s'abstraire des frontières nationales considérées comme trop exiguës. II n'est donc pas surprenant de constater qu'un peu plus de $10 \%$ des managers allemands détiennent un MBA et plus de $12 \%$ de leurs homologues français.

Pays d'origine des dirigeants étrangers dans les entreprises du DAX 30 et du CAC 40 en 2006

\begin{tabular}{|c|c|c|c|c|c|}
\hline Pays d'origine & DAX 30 & CAC 40 & Pays d'origine & DAX 30 & CAC 40 \\
\hline Afrique du Sud & 1 & - & Italie & 2 & 11 \\
\hline Allemagne & - & 8 & Luxembourg & - & 2 \\
\hline Argentine & 1 & - & Nouvelle-Zélande & 2 & 1 \\
\hline Australie & - & 1 & Pays-Bas & 2 & 2 \\
\hline Autriche & 5 & 2 & Portugal & - & 1 \\
\hline Belgique & 1 & 11 & Suède & 1 & - \\
\hline Canada & 1 & 2 & Suisse & 1 & 2 \\
\hline Danemark & 1 & - & USA & 10 & 20 \\
\hline Espagne & 2 & 4 & & & \\
\hline Finlande & - & 2 & Total & 39 & 80 \\
\hline France & 1 & - & Nationalités connues & 192 & 319 \\
\hline Grande-Bretagne & 4 & 7 & $\%$ d'étrangers & $20,31 \%$ & $25,08 \%$ \\
\hline Grèce & 1 & - & - dont Européens & 21 sur 39 & 52 sur 80 \\
\hline Inde & - & 4 & soit en $\%$ des étrangers & $53,85 \%$ & $65,00 \%$ \\
\hline Iran & 1 & - & - dont anglo-saxons & 13 sur 39 & 24 sur 80 \\
\hline Israël & 2 & - & soit en $\%$ des étrangers & $33,33 \%$ & $30,00 \%$ \\
\hline
\end{tabular}

Source : nos propres calculs d'après les rapports annuels des entreprises pour 2006.

Internationalisation de leur recrutement
Cette internationalisation des formations s'accompagne, en France comme en Allemagne, d'une internationalisation du recrutement. Plus de $20 \%$ des dirigeants des entreprises du DAX 30 sont de nationalité étrangère, et c'est le cas de $25 \%$ des dirigeants des sociétés du CAC 40. Si de nombreuses nationalités sont représentées, il est à noter que cette internationalisation est majoritairement européenne, et que les dirigeants anglo-saxons sont proportionnellement aussi nombreux des deux côtés du Rhin. Ils représentent 33 \% des étrangers dans les entreprises françaises et $30 \%$ dans les entreprises allemandes. Cependant, toutes les grandes entreprises n'ouvrent pas leur direction aux étrangers : dans celles du CAC 40, 7 sur 30 (soit environ $23 \%$ ) ne recrutent que des Français, et c'est notamment le cas de banques (BNP-Paribas, Crédit Agricole, Société Générale), en dépit de l'ouverture internationale des échanges de capitaux. En Allemagne, 7 entreprises du DAX 30 (soit $23 \%$ ) ne recrutent que des dirigeants allemands pour intégrer leur directoire. Cette internationalisation des équipes dirigeantes 
présente, on le voit, de grandes similitudes en France et en Allemagne : elle a la même ampleur et revêt une fonction symbolique analogue. On notera au passage que si on dénombre 8 dirigeants de nationalité allemande dans les sociétés du CAC 40 (dont 4, il est vrai, chez EADS), un seul Français est membre d'un directoire dans celles du DAX 30 (J.-P. Thierry chez Allianz).

\section{Présentation méthodologique}

Pour observer dans quelle mesure ces mutations de l'environnement économique ont modifié les caractéristiques des profils et parcours des dirigeants exposées dans la littérature, nous avons procédé à une étude empirique. Nous avons construit pour ce faire une base de données sur les 424 dirigeants (du Comité exécutif) des entreprises cotées au CAC 40 et sur les 192 dirigeants des sociétés cotées au DAX 30, en 2006. Les données utilisées proviennent pour l'essentiel des documents publiés par les entreprises elles-mêmes (notamment dans le rapport annuel). Elles portent sur l'âge des dirigeants, leur nationalité, leur formation et les diplômes obtenus, leur parcours professionnel avec notamment la date de nomination dans le comité exécutif ou directoire. A partir de ces données, des statistiques descriptives ont été réalisées.

Les mutations de l'environnement économique des entreprises affectent aussi les systèmes de formation des élites économiques. Dans leur recrutement des futurs dirigeants, les entreprises allemandes continuent d'entériner les sélections opérées par le système d'enseignement supérieur universitaire : moins de $5 \%$ des dirigeants des sociétés du DAX 30 n'ont que le bac ou un niveau bac. La détention d'un diplôme d'enseignement supérieur demeure donc presque indispensable pour accéder aux fonctions de direction.

La création, dans les années 1960-70, d'universités nouvelles et de 'l'université de masse' n'a pas remis en cause l'absence de hiérarchie entre les universités. Aucune université ne se distingue comme vivier particulier de dirigeants. De même constate-t-on la persistance de la grande variété des formations universitaires avec une représentation relativement forte des formations d'ingénieurs disposant d'une expertise dans une discipline (physique, chimie, construction mécanique... ; près de $20 \%$ ) et de juristes (12\%). Comme par le passé, les grandes entreprises privilégient les détenteurs d'un titre de docteur (plus de $34 \%$ ), quel qu'il soit. A maints égards, les grandes entreprises allemandes continuent donc de se plier à l'exigence de 'légitimité académique' dans leur recrutement.

\section{Allemagne :}

persistance du socle

institutionnel de formation, ...

Les diplômes détenus par les dirigeants des entreprises cotées au DAX 30 en 2006

\begin{tabular}{|lrr|}
\hline \multicolumn{1}{|c}{$\quad$ Diplômes et formations } & Nombre & \% du total connu (192) \\
Doct. droit - sciences juridiques & 9 & 4,69 \\
Doct. sciences sociales, politiques, philosophie & 11 & 5,73 \\
Doct. sciences de l'ingénieur & 11 & 5,73 \\
Doct. physique & 6 & 3,13 \\
Doct. chimie, biologie & 11 & 5,73 \\
Doct. construction mécanique, électrotechnique & 3 & 1,56 \\
Doct. sciences économiques & 9 & 4,69 \\
Doct. mathématiques & 5 & 2,60 \\
MBA - INSEAD & 20 & 10,42 \\
Etudes de droit, avocat & 14 & 7,29 \\
Etudes univ. économie-gestion (Bac+4 et +3) & 72 & 37,50 \\
Etudes univ. constr. mécanique, électronique, électrotechnique, aéronavale (Bac+4 et +3) & 23 & 11,98 \\
Etudes univ. mathématiques, physique, chimie (Bac+4) & 12 & 6,25 \\
Autres diplômes d'université (informatique, lettres, langues) & 20 & 1 \\
Bact2 & 10,42 \\
Moins que Bac et Bac & 9,52 \\
Apprentissage & 4,69 & 7,29 \\
\hline
\end{tabular}

Source : nos propres calculs.

Cette persistance des caractéristiques majeures, endogènes au modèle de for... mais percée du MBA... mation allemand, n'exclut pas des changements substantiels qui indiquent que des évolutions fondamentales sont en cours. Parmi ces changements figure l'importance nouvelle des formations universitaires en économie et gestion $(\mathrm{Bac}+3$ et surtout +4 , mais sans conclusion par un doctorat) comme voie d'accès principale aux fonctions de direction. Plus de $37 \%$ des managers du DAX 30 ont suivi ce parcours. On peut sans doute déceler dans cette tendance une nouvelle exi- 
... et perte de vitesse de l'apprentissage

Recul des docteurs en droit

France : persistance des parcours d'excellence traditionnels

Les grandes écoles d'ingénieurs toujours en tête gence des entreprises : les dirigeants doivent maîtriser les outils de gestion et disposer d'une vision globale du fonctionnement et de l'organisation de l'entreprise. C'est aussi l'une des formations qui permet de discuter 'entre pairs' avec les commissaires aux comptes, analystes financiers des agences de notation internationales.

En 2006, l'apprentissage ne paraît plus jouir des mêmes faveurs et de la même valorisation dans la grande entreprise allemande. Les dirigeants des sociétés du DAX 30 en 2006 sont moins de $10 \%$ à avoir suivi cette voie, ce qui dénote une perte de reconnaissance de la formation acquise au sein de l'entreprise au profit des diplômes initiaux. En outre, l'âge relativement jeune dans la fonction d'un certain nombre de managers (près de $20 \%$ des dirigeants ont moins de 46 ans) ne permet pas de penser qu'ils ont gravi les échelons de la hiérarchie avant d'accéder aux plus hautes fonctions dirigeantes. Leur parcours dans l'entreprise ne permet pas non plus de déceler des réussites exceptionnelles qui les auraient propulsés à ces postes.

Derrière l'indéniable stabilité de leur préférence pour les titulaires d'un doctorat, les entreprises procèdent à des choix qui induisent des changements et d'autres distributions en fonction des disciplines. Si jusqu'alors, les titulaires d'un doctorat de droit jouissaient d'une suprématie incontestée, les docteurs en sciences économiques ou de gestion sont aussi nombreux en 2006 : ils représentent eux aussi près de $5 \%$ de la population totale des dirigeants, et le groupe des dirigeants titulaires d'un doctorat des sciences sociales et politiques est même plus nombreux (près de $6 \%$ ). L'analyse des influences des différentes formations, au moyen des outils d'analyse des réseaux sociaux, corrobore les constats précédents (Massol/ Vallée, 2010). Néanmoins, cette approche relativise sensiblement l'importance du diplôme de docteur par rapport à celui de MBA. Elle montre notamment que, bien que le nombre des MBA soit trois fois moins élevé que celui des doctorats, le diplôme MBA occupe une position plus centrale dans le réseau des formations et est donc susceptible d'être plus influent. En dépit de la stabilité dans le temps du modèle national de formation et de recrutement, force est de constater que les nouvelles caractéristiques distinctives des profils de dirigeants allemands indiquent que le management rhénan s'adapte à son environnement mondialisé.

Le recrutement des dirigeants par les grandes entreprises françaises est d'abord caractérisé par une continuité des exigences et des règles souvent tacites qui président à leurs choix. Comme en Allemagne, les grandes entreprises choisissent plus que jamais des dirigeants diplômés de l'enseignement supérieur : ils étaient plus de $80 \%$ en 1994, ils sont $99 \%$ en 2006. En revanche, en France, être diplômé signifie dans $65 \%$ des cas être diplômé d'une grande école et non posséder un titre universitaire. Comme pendant les années 1980 et 1990, on constate la suprématie de trois grandes écoles (Polytechnique, ENA, HEC) qui paraissent une voie royale vers les postes de dirigeants : près de $50 \%$ du total des dirigeants des entreprises du CAC 40 en 2006 ont fréquenté ces écoles.

A l'intérieur du groupe des grandes écoles, on retrouve les mêmes distributions qu'au cours des deux décennies précédentes : les grandes écoles formant des ingénieurs ( $X$, Mines, Ponts, Centrale, ENSAE) constituent près de $31 \%$ du vivier des dirigeants du CAC 40, et les grandes écoles formant à la gestion et au management (HEC, ESSEC, ESCP) près de $18 \%$. Toutes les autres écoles d'ingénieurs forment un nombre de dirigeants qui est près de 2,5 fois moins élevé (12\%), alors que l'effectif total de ces dernières est sensiblement plus nombreux. Il en va de même pour les autres formations à la gestion et au management qui ne forment qu'à peine $6 \%$ des dirigeants.

Il est à noter que la distinction traditionnelle grandes écoles d'ingénieurs versus grandes écoles de gestion-management masque de fortes disparités à l'intérieur de ces grands groupes. L'X est le plus important vivier de dirigeants avec 16,2\% des dirigeants formés, alors que l'Ecole centrale ne représente que 2,7\% des effectifs, tout en ayant des tailles de promotions comparables à celles de l'X. Parmi les écoles de gestion-management, on retrouve la même prééminence d'une 
école : HEC qui, avec 10,2 \%, est loin devant l'ESSEC (5,2\%) et l'ESCP (2,2 \%). L'ENA continue de constituer un tremplin vers les fonctions de dirigeant $(11 \%$ des dirigeants français), souvent après quelques années passées dans l'administration centrale ou dans un ministère.

Les diplômes détenus par les dirigeants des entreprises cotées au CAC 40 (2006)

\begin{tabular}{|lcc|}
\hline Diplômes & Nombre & \% du total connu (402) \\
ENA & 43 & $10,70 \%$ \\
Polytechnique & 65 & $16,17 \%$ \\
Centrale - Mines - Ponts & 49 & $12,19 \%$ \\
IEP & 47 & $11,69 \%$ \\
ENS & 11 & $2,74 \%$ \\
ENSAE - ENSTelecom & 9 & $2,24 \%$ \\
HEC - ESCP - ESSEC & 71 & $17,66 \%$ \\
Autres écoles de commerce & 23 & $5,72 \%$ \\
Autres écoles d'ingénieurs & 50 & $12,44 \%$ \\
MBA - INSEAD - CPA - IEDC & 51 & $12,69 \%$ \\
Université - Doctorat & 39 & $9,70 \%$ \\
Autres diplômes universitaires & 164 & $40,80 \%$ \\
Bac +2 & 7 & $1,74 \%$ \\
Bac et moins que Bac & 4 & $1,00 \%$ \\
\hline
\end{tabular}

Source : nos propres calculs. Le total ne s'élève pas à $100 \%$ en raison des doubles diplômes.

S'il est vrai que trois grandes écoles forment traditionnellement plus de la moitié des dirigeants des grandes entreprises françaises, il faut souligner que cette proportion a baissé sensiblement: de $57 \%$ en 1993, elle est passée à moins de $50 \%$ en 2006. Cette baisse générale occulte, ici encore, des évolutions contradictoires : I'X connaît une baisse (près de 4 points), l'ENA une baisse plus sensible encore (plus de 8 points), tandis qu'HEC forme davantage de dirigeants (d'environ $7 \%$ à plus de $13 \%$ ). Parmi les établissements d'enseignement supérieur formant des dirigeants, on voit apparaître la concurrence de l'Institut d'Etudes Politiques de Paris (IEP, près de $12 \%$ des dirigeants y ont été formés). Peut-être faut-il déceler ici les premières conséquences de l'internationalisation de cette formation qui fonctionne de moins en moins comme une classe préparatoire à l'ENA que comme une grande école à part entière. Une analyse par les outils des réseaux sociaux montre que I'X est plus influente que l'ENA et qu'effectivement l'IEP occupe une place privilégiée (Massol/Vallée, 2010).

Sans que soit remise en cause la suprématie des diplômés des grandes écoles, on peut déceler quelques signes d'ouverture des grandes entreprises françaises vers une certaine variété des diplômes universitaires qui, même si elle n'est pas comparable à la diversité allemande, n'est pas pour autant négligeable. II n'est en tout cas plus possible d'affirmer comme dans les années 1980 et 1990, qu'en dehors de quelques grandes écoles, peu d'établissements d'enseignement supérieur jouent un rôle significatif dans la formation des dirigeants. Si l'université formait $10 \%$ des dirigeants des 'Top 200' en 1985, $9 \%$ en 1989 et seulement $4 \%$ en 1993, la tendance s'est inversée : près de $19 \%$ des dirigeants des grandes entreprises françaises sont passés par l'université, et $10 \%$ des managers ont un titre de docteur.

EN 2006, LE MODE DE FABRICATION ET DE SÉLECTION DES DIRIGEANTS des plus grandes entreprises allemandes et françaises demeure fondamentalement stable et différent. En France, la sélection continue de reposer très largement sur des critères de réussite à des concours scolaires organisés au niveau Bac +2 et qui donnent accès à des grandes écoles généralistes, imposant leur suprématie dans la constitution de viviers des futurs dirigeants. Ce mode de sélection laisse de facto peu de place à la formation initiale dispensée par l'université et reproduit très largement des hiérarchies culturelles et sociales. En Allemagne, la sélection des élites économiques intervient plus tardivement, à la fin de parcours universitaires qui se caractérisent d'abord par leur grande diversité et qui sont accessibles à tous $(\mathrm{Bac}+4)$, souvent après un doctorat. La voie de l'apprentissage et la promotion interne jouent encore un rôle non négligeable.
L'importance relative des établissements respectifs est en train de changer

Une plus grande variété des formations s'esquisse 
II est indéniable que la globalisation, l'internationalisation des firmes et de leur environnement économique ne sont pas restées sans effet sur ces modèles nationaux de production et de sélection des élites économiques. Ces dernières sont devenues plus internationales, davantage imprégnées de la culture d'entreprise anglo-saxonne qui impose ses règles de fonctionnement aux marchés, aux organisations mondialisés.

Pourtant, ces puissantes logiques économiques et sociales n'ont pas eu raison des particularités nationales majeures, elles n'ont pas standardisé les parcours de formation des élites économiques françaises et allemandes. L'engouement pour le MBA en France et en Allemagne n'a nullement estompé les différences profondes des modèles nationaux de formation. II est d'ailleurs possible que, contre toute attente, le caractère plus monolithique du système de formation français permette une diffusion plus rapide et une adoption moins critique de la culture d'entreprise anglo-saxonne. C'est du moins ce que laisse penser l'empressement avec lequel les dirigeants français ont, dès 1995, accueilli les 'meilleures pratiques' de gouvernance d'entreprise diffusées par le monde anglo-saxon, alors qu'il a fallu attendre le début des années 2000 en Allemagne pour y voir apparaître les premiers codes de bonne gouvernance. Le profond ancrage, dans la culture allemande, du modèle de la stakeholder value et de son orientation sur le long terme, de même que la grande diversité observée dans le recrutement des élites, peu propice par essence à l'émergence d'un mainstream, rendaient peutêtre moins facile l'application quasi mimétique de 'meilleures pratiques' de gouvernance dont l'efficience supérieure déclarée par ses promoteurs reste encore à démontrer.

\section{Indications bibliographiques}

-Abelshauser W., Der deutsche Weg in die Neue Wirtschaft und die amerikanische Herausforderung, Berlin, 2003

- Bauer M., Bertin-Mourot B., « La tyrannie du diplôme initial et la circulation des élites : la stabilité du modèle français », in Suleiman E., MENDRAS H. (dir.), Le recrutement des élites en Europe, Paris, 1995

- BAUER M., Bertin-Mourot B., Vers un modèle européen de dirigeants ? Ou trois modèles contrastés de production de l'autorité légitime au sommet des grandes entreprises? Comparaison France / Allemagne / Grande-Bretagne, Paris, 1996

- CNUCED, World Investment Report, 1997, 2008

- D'IRIBARNE P., Cultures et mondialisation, Paris, 1998

-DUdouet F.-X., Gremont E., " Les grands patrons et l'Etat en France. 1981-2007 ", in Sociétés contemporaines, $\mathrm{n}^{\circ}$ 68, 2007

-Ehrenberg A., Le culte de la performance, Paris, 1991

HOFSTEDE G., Culture's Consequences : international differences in work-related values, Londres, 1980

- HARTMANn M., Der Mythos von den Leistungseliten. Spitzenkarrieren und soziale Herkunft in Wirtschaft, Politik, Justiz und Wissenschaft, Francfort/Main, 2002

- HARTMANN M., « Le recrutement des dirigeants des grandes entreprises en Allemagne. Une sélection sociale en l'absence d'institutions de formation des élites », in JoLY H. (dir.), Formation des élites en France et en Allemagne, Coll. Travaux et documents du CIRAC, Cergy-Pontoise, 2005

- HARTMANN M., Eliten und Macht in Europa. Ein internationaler Vergleich, Francfort/Main, 2007

- Joly H., "Concours des grandes écoles versus diplômes universitaires. Sélectivité comparée des élites économiques françaises et allemandes ", in Joly H. (dir.), Formation des élites en France et en Allemagne, Coll. Travaux et documents du CIRAC, Cergy-Pontoise, 2005a

- Joly H., « Les élites politiques : regard croisé sur le cas français », in Joly H. (dir.), Formation des élites en France et en Allemagne, Coll. Travaux et documents du CIRAC, Cergy-Pontoise, 2005b

- MASSOL J., VALLÉE T., « La gouvernance d'entreprise en France et en Allemagne : l'impact de la mondialisation », in BRÉMOND J., MASSOL J. (dir.), Cultures nationales et mondialisation, Nantes, 2008

- MASSol J., VALLÉE T., "Les réseaux sociaux des formations des dirigeants des entreprises du CAC 40 et du DAX 30 ", document de travail du LEMNA, Nantes, 2010

- MucchielLi J.-L., Multinationales et mondialisation, Paris, 1998

- REISACH U., Die Amerikanisierungsfalle. Kulturkampf in deutschen Unternehmen, Berlin, 2007

- ReISACH U., "Culture d'entreprise : 'le piège de l'américanisation' », entretien, Regards sur l'économie allemande, $n^{\circ} 84$, décembre 2007

- Suleiman E., Mendras H. (dir.), Le recrutement des élites en Europe, Paris, 1995

- van Zanten A. (dir.), Dictionnaire de l'éducation, Paris, 2008

-WAGNER A.-C., Les classes sociales dans la mondialisation, Paris, 2007. 